\title{
Liliput oder Leviathan? Der Staat in der globalisierten Wirtschaft
}

\author{
Frey, Bruno S
}

\begin{abstract}
Globalization is often seen to result in a smaller (Lilliput) or larger (Leviathan) state. But future public activity will be more flexible. People have multiple identities. They can be citizens of suband supra-national jurisdictions, semi- and non-governmental organizations and private units, even profitoriented firms. Such attachment may be temporary, multiple or partial. To actively choose strengthens loyalty and identification, which raises the willingness to pay for publicly supplied services (in the sense of quasi-voluntary taxation). On the supply side, Functional, Overlapping and Competing Jurisdictions (FOCJ) will develop. Such flexibility of future European integration will make it successful.
\end{abstract}

DOI: https://doi.org/10.1111/1468-2516.00099

Posted at the Zurich Open Repository and Archive, University of Zurich

ZORA URL: https://doi.org/10.5167/uzh-155360

Journal Article

Published Version

Originally published at:

Frey, Bruno S (2002). Liliput oder Leviathan? Der Staat in der globalisierten Wirtschaft. Perspektiven der Wirtschaftspolitik:n/a.

DOI: https://doi.org/10.1111/1468-2516.00099 


\title{
Liliput oder Leviathan? Der Staat in der globalisierten Wirtschaft
}

\author{
Bruno S. Frey* \\ Universität Zürich
}

\section{Was wird aus dem Staat?}

Die Debatte über die Zukunft des Staates angesichts der Globalisierung ${ }^{1}$ wird durch zwei völlig gegensätzliche Auffassungen geprägt.

\section{Der Liliput-Staat}

Ein Szenario prophezeit ein Verschwinden des Staates; zumindest büßt er wesentlich an Größe ein und wird zum Liliput. Öffentliche Güter, der Sozialstaat und die Umverteilung lassen sich nicht mehr aufrechterhalten. Es kommt zu sozialem Dumping. Weitergehender wird sogar der Untergang der Demokratie und Freiheit vorausgesehen (z.B. Barber 1995). Die entstehenden Probleme äußern sich bereits in Form der weltweiten und oft gewalttätigen Proteste gegen die Globalisierung.

Das „race to the bottom“ und die „zero regulation“ werden als Auswirkungen eines ungeregelten Wettbewerbs der Systeme und insbesondere des Steuerwettbewerbs gesehen. ${ }^{2}$ Gemäß dem Hauptergebnis der traditionellen Theorie des Steuerwettbewerbs sind die Steuersätze im Nash-Gleichgewicht für eine effiziente Finanzierung öffentlich angebotener Konsumgüter zu tief. Je stärker überdies die Spillovers zwischen den Staaten sind, desto intensiver wird

* Prof. Dr. Bruno S. Frey, Institut für Empirische Wirtschaftsforschung, Universität Zürich, Bluemlisalpstr. 10, CH-8006 Zürich, Tel +41 1 6343730, Fax +41 1 6344907, E-Mail: (bsfrey@iew.unizh.ch). Für wertvolle Verbesserungsvorschläge danke ich meinen Mitarbeitern und vielen Kollegen. Aus Platzgründen können nur wenige Literaturhinweise gegeben werden. Sie finden sich in der erweiterten Fassung als Working Paper des Institut für Empirische Wirtschaftsforschung, Universität Zürich; http://www.unizh.ch/iew/grp/frey

1. Zur Globalisierung vgl. z.B. Bernholz (2000), Bernauer (2000), Kirchgässner (1998), Rodrik (1997). Eine hervorragende und ausführliche Darstellung der quantitativen Aspekte geben Schulze und Ursprung (1999).

2. Für Übersichten vgl. Straubhaar (1998), Schaltegger und R.L. Frey (2001), Feld (2000a,b), Wilson (1999).

(C) Verein für Socialpolitik und Blackwell Publishers Ltd. 2002, 108 Cowley Road, Oxford OX4 1JF, UK und 350 Main Street, Malden, MA 02148, USA. 
Trittbrett gefahren und desto ausgeprägter ist das Unterangebot an öffentlichen Gütern. Die Regierungen senken die Steuerbelastung, um heimatloses Kapital und global mobile Personen anzuziehen. Als spezifische Ursachen für das "Verschwinden des Steuerzahlers" (Economist 2000) werden vor allem genannt die Elektronisierung des Handels, der Transaktionen und des Geldes; zunehmende Transaktionen innerhalb globaler Firmen; Steuerparadiese („offshore financial centers"); Derivative und Hedge Funds; die grenzenlose Mobilität des finanziellen Kapitals und zunehmende Aktivitäten außerhalb des eigenen Landes (vgl. Tanzi 1996). ${ }^{3}$

\section{Der Leviathan-Staat}

Ein entgegengesetztes Szenario wird ebenso häufig vertreten. Der Staat ist eine uralte und überaus mächtige Institution, die immer zu überleben weiß. Die Politiker und Bürokraten sind bisher noch immer fähig gewesen, neue Möglichkeiten der Besteuerung auszudenken und praktisch umzusetzen. Als Reaktion auf den drohenden Verlust der Besteuerungsgrundlagen infolge der Globalisierung werden sie eine „Harmonisierung", d.h. eine Gleichschaltung, der Steuern, durchsetzen. Manche befürworten sogar eine „World Tax Organization", die den Staaten überall auf der Welt einen Zugriff auf die Steuerzahler ermöglicht. Daneben werden mannigfaltige Regulierungskartelle treten (Vaubel 2000: 283 gibt eine ausführliche Liste der Möglichkeiten). Weil der Wettbewerb zwischen den Staaten unterdrückt wird, stehen die einzelnen Bürger dann einem Leviathan gegenüber, dem sie immer stärker ausgeliefert sind.

Auch Rodrik (1998) verbindet Globalisierung mit einem größeren Staat. Nach dem zweiten Weltkrieg wurde ein impliziter Gesellschaftsvertrag geschlossen, wonach die Verlierer der Globalisierung durch fiskalpolitische Programme kompensiert werden. Gemäß der Neuen Ökonomischen Geografie (Baldwin und Krugman 2001) bewirken Agglomerationskräfte, dass selbst vollständig mobiles Kapital zu einem quasi-fixen Faktor wird, wodurch das "race to the bottom“ verhindert wird. Die Länder mit einem guten Angebot an Infrastruktur (inklusive Rechtssicherheit) können als Monopolisten einen Limit-Preis erheben. Die Globalisierung bewirkt dann nicht zwingend fallende Steuersätze und ist mit einem großen Wohlfahrtsstaat völlig vereinbar.

\section{Thesen}

Auf dem Hintergrund dieser divergierenden Vorstellungen über die Zukunft des Staates in einer globalisierten Gesellschaft werden hier vier Thesen vertreten:

3. Die Vorstellung eines ruinösen Wettbewerbs wird im deutschsprachigen Raum z.B. von Sinn (1999) oder Janeba (1997) vertreten; vgl. differenziert auch Richter (2001). Dagegen wenden sich jedoch z.B. Blankart (2001, 2002), Siebert (1998), Siebert und Koop (1993), Vanberg und Kerber (1994), Berthold und Naumann (2001), Fehn (2001). 


\section{Liliput oder Leviathan?}

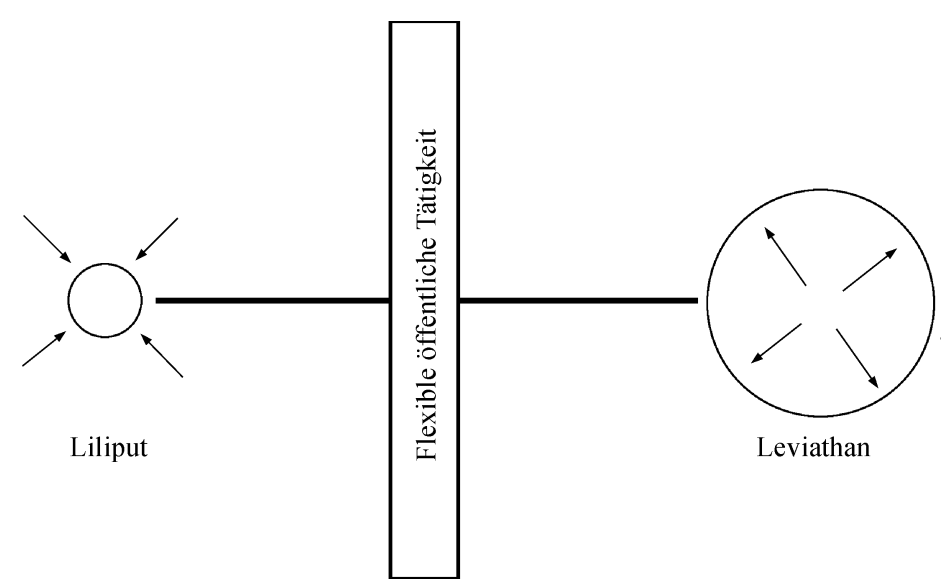

Abbildung 1 Dimensionen der Staatengröße

1. Die öffentliche Tätigkeit wird zukünftig völlig anders als heute sein. Der Staat, mit dem wir vertraut sind, wird verschwinden - und ist teilweise auch schon verschwunden. Aber es wird auch kein Weltstaat mit Zwangsgewalt entstehen. Aus diesen Gründen ist die Konzentration auf die zu erwartende Größe des Staates belanglos. Abbildung 1 zeigt, wie die bisher diskutierte Dimension der Größe mit dem Liliput-Staat auf der einen, und dem Leviathan-Staat auf der andern Seite, durch eine Diskussion der möglichen zukünftigen neuen Formen abgelöst werden sollte.

2. Die zukünftige öffentliche Tätigkeit wird durch flexible, multiple und temporäre Körperschaften unternommen.

3. Die klassische monopolistische Beziehung zwischen Bürger und Nationalstaat weicht sich auf. Entsprechend wird auch die Staatszugehörigkeit multipel, temporär und partiell sein.

4. Es wird möglich sein, Bürger unterschiedlichster Organisationen zu werden: neben subnationalen und supranationalen staatlichen Körperschaften auch bei halbstaatlichen, nicht-staatlichen und privaten Organisationen und sogar bei globalen Firmen.

\section{Konsequenzen}

Erweisen sich diese Thesen als richtig, ergeben sich schwerwiegende Auswirkungen auf die Wirtschaft und Gesellschaft, aber auch auf die Wirtschaftstheorie. Sie werden im Verlauf dieser Arbeit behandelt, sollen hier aber bereits angedeutet werden:

1. Mannigfache Identitäten werden für die Menschen das Normale sein; sie werden vielerlei Zugehörigkeiten und Loyalitäten haben.

Der Nationalstaat als dominante Institution des 19. und 20. Jahrhunderts, büßt seine Monopolstellung ein. 
2. Die bisher dem Nationalstaat vorbehaltene Identität und Loyalität und damit auch gefühlsmäßige "Wärme" wird in andere Bereiche, bisher als „kalt" perzipierte Bereiche (wie den Markt) übertragen.

3. Die Dichotomie zwischen "Staat" und „Markt" ist endgültig vorbei; vielmehr müssen die mannigfachen öffentlichen Einheiten untersucht werden.

\section{Vorgehen}

Im folgenden Abschnitt wird nachfrageseitig ein neuartiges Konzept der Bürger und in Abschnitt 3 angebotsseitig neue Möglichkeiten staatlicher Organisation vorgestellt. In Abschnitt 4 wird die Möglichkeit mannigfacher Identitäten weiter ausgeführt. Im letzten Abschnitt werden die Hauptergebnisse zusammengefasst.

\section{Bürger in der globalen Welt}

Die Individuen werden sich in der Zukunft vom Monopolanspruch des Nationalstaats befreien und aktiv eine Wahl treffen können, wessen Bürger ${ }^{4}$ sie sein möchten. Bis heute definieren die Nationen im wesentlichen von sich aus, wer zu ihren Bürgern zählt. Die Individuen können nur sehr begrenzt über den Eintritt und Austritt selbst entscheiden. Vor allem bezieht sich die Bürgerschaft traditionell ausschließlich auf den Staat; andere Zugehörigkeiten werden zum vorneherein ausgeschlossen.

Der Nationalstaat als einzige Identität des Bürgers ist in der globalen Gesellschaft überholt, weil diese durch eine ausgeprägte Heterogenität in zweierlei Hinsicht charakterisiert ist:

(a) Die Präferenzen der Individuen unterscheiden sich wesentlich voneinander; sie lassen sich nicht (mehr) allein durch die als homogen gedachte Institution der Nation erfüllen.

(b) In einer globalen Gesellschaft fühlt sich ein Individuum in aller Regel vielen Organisationen zugehörig; die Nation allein kann auch dieser Präferenz nicht gerecht werden.

Individuen können deshalb Bürger auch von Organisationen außerhalb der Nation sein. Folgende Möglichkeiten lassen sich denken:

1. Individuen sind Bürger sub-nationaler Körperschaften wie Regionen, Provinzen oder Gemeinden, oder aber supra-nationaler Körperschaften wie der Europäischen Union, der NATO, der Weltbank ${ }^{5}$ oder der UNO.

2. Personen sind Bürger auch in halb-staatlichen Organisationen. Dafür kommen viele verschiedene Institutionen in Frage. Ein Beispiel sind Universitäten.

4. Ausschließlich aus Gründen der sprachlichen Vereinfachung wird hier der Begriff „Bürger“ verwendet; es sind damit auch Bürgerinnen gemeint.

5. Angehörige der Weltbank reisen bereits heute teilweise mit einem entsprechenden Pass. (Private Mitteilung von Dennis Mueller.) 


\section{Liliput oder Leviathan?}

Tatsächlich hat im deutschsprachigen Raum der Begriff des „Universitätsbürger" eine alte, aber weitgehend verloren gegangene Tradition. Sie ist hingegen in den Vereinigten Staaten sehr lebendig. Ein amerikanischer Alumnus gibt sein Geld nicht derjenigen Organisation, die den höchsten Nutzen daraus zieht, sondern „... contributions may well reflect identity. Graduates give to their own alma mater" (Akerlof und Kranton 2000: 722).

3. Personen sind Bürger nicht-staatlicher Organisationen (Non-Governmental Institutions, NGOs). Beispiele sind global tätige karitative Organisationen wie das Rote Kreuz oder die Médecins sans Frontières.

4. Personen sind Bürger privater Organisationen. Dazu gehören globale funktional orientierte Vereinigungen wie z.B. ICANN (die Internet Co-operation for Assigned Names and Numbers, vgl. dazu Engel und Keller 2000), sportliche (wie die FIFA), künstlerische oder wissenschaftliche Vereinigungen. Auch international orientierte Clubs wie Bayern München oder Real Madrid können Personen als Bürger haben, wenn diese ihnen besonders nahe stehen und bereit sind, auch entsprechende Pflichten (insbesondere ein Wohlverhalten innerhalb und außerhalb der Stadien) zu übernehmen. Viele Personen verfügen über soviel Identität und Loyalität, dass sie derartigen privaten Organisationen freiwillig erhebliche Geldbeiträge für die von ihnen angebotenen öffentlichen Leistungen zukommen lassen (selbstverständlich sind diese auch auf andere Einnahmen angewiesen).

5. Individuen sind Bürger gewinnorientierter Firmen. In der Betriebswirtschaftslehre wird von „organizational citizenship“ (z.B. Organ 1988, van Dyne, Graham und Dienesch 1994) gesprochen. Große Bedeutung wird insbesondere der „corporate citizenship“ zugewiesen. Darunter wird das nicht durch Vorschriften erzwungene und über vertragliche Verpflichtungen hinausgehende Verhalten im Interesse der Firma verstanden. Bürgertum ist vor allem für global tätige Firmen mit einem über die gesamte Welt verstreuten Filialnetz geeignet. Diese können für ihre Bürger viele Aspekte regeln und übernehmen, die sich bisher Nationalstaaten vorbehalten haben, wie etwa eine Arbeitserlaubnis einzuholen, finanzielle Garantien zu geben und Identifikationskarten (Pässe) auszustellen. Entscheidend ist die spezielle Loyalitätsbeziehung des Bürgers zu "seiner“ Firma. Diese geht wesentlich über ein Aktionärs-, Kunden- oder Beschäftigtenverhältnis hinaus. Wer nur mit Aktien spekuliert, ist kein Bürger der entsprechenden Firma. Bürger kann nur werden, wer ein Grundvertrauen $\mathrm{zu}$,seiner" Firma hat. Er behält seine Aktien auch dann, wenn es der Firma schlecht geht und Spekulanten längst verkauft haben. Bürger sind auch nicht nur Stakeholder, denn diese verfügen nicht über formale demokratische Mitwirkungsmöglichkeiten wie sie Bürger besitzen. ${ }^{6}$

6. Wie die deutsche Mitbestimmung zeigt, ist es durchaus möglich, die verschiedenen Funktionen zu kombinieren. So braucht zum Beispiel nicht jeder Beschäftigte auch ein Bürger einer Firma zu sein, und umgekehrt kann jemand Bürger einer Firma sein, ohne dort beschäftigt zu sein oder Aktien zu besitzen. 
In der Zukunft wird der Begriff des Bürgers flexibel gehandhabt werden (vgl. auch Tullock 1997). Insbesondere sind folgende Varianten vorstellbar:

- Temporäre Bürgerschaft. Eine Person soll wählen können, für welche Periode sie sich in der erforderlichen engen Weise mit einer bestimmten Organisation verbinden will;

- Multiple Bürgerschaft. Jedermann kann gleichzeitig Bürger verschiedener Organisationen sein;

- Partielle Bürgerschaft. Eine Person kann Bürger nur eines Teils einer Organisation, wie z.B. der Sozialversicherung eines bestimmten Staates sein.

Die Idee der Bürgerschaft wird somit wesentlich erweitert und geht weit über die alleinige Beziehung von Personen zum Nationalstaat hinaus. Daraus wird die spezielle Eigenschaft des erweiterten Bürgerkonzeptes deutlich: Es handelt sich um einen unvollständigen und freiwilligen Vertrag zwischen Individuen und von ihnen gewählten Organisationen. Der Inhalt des Vertrags lässt sich nicht a priori festschreiben. Aus diesem Grund ist neben den jeweiligen spezifischen Rechten (insbesondere die Möglichkeit der Beteiligung im Entscheidungsprozess) und Pflichten (insbesondere sich an die beschlossenen Regeln zu halten) die intrinsische Motivation für die Bürgerschaft von essentieller Bedeutung. Intrinsische Motivation äußert sich in Form von Loyalität, Identifikation und Engagement, die gerade dann wirksam werden, wenn der Vertrag nichts oder zu wenig aussagt. Intrinsische Motivation steht im Gegensatz zur extrinsischen Motivation, bei der Menschen auf Grund äußerer (oft monetärer) Anreize für sich selbst Nutzen und Kosten abwägen. Intrinsische Motivation ist in der Ökonomik lange vernachlässigt worden, weil sie als theoriefremd angesehen wurde. In jüngster Zeit hat sich die Situation jedoch drastisch geändert. Aspekte wie Vertrauen, Sozialkapital, Fairness, Reziprozität, Identifikation oder Loyalität $^{7}$ werden heute in die Wirtschaftstheorie integriert (z.B. Akerlof und Kranton 2000, Loewenstein 2000, Fehr und Gächter 2000, Frey 2001; allgemein Bowles 1998).

Die durch die Bürgerschaft begründete besondere Beziehung zwischen Individuen und Organisationen übt zweierlei Wirkungen aus:

(a) Bestehende intrinsische Motivation wird auf bestimmte Organisationen kanalisiert und die Identifikation damit gefestigt. Die betreffende Organisation übernimmt damit eine „expressive“ Funktion (vgl. Jones und Hudson 2000) und wirkt normverstärkend. ${ }^{8}$

(b) Potentielle intrinsische Motivation wird aktiviert und Bürgertugenden werden geweckt. Damit werden auch andere Personen zu Beiträgen zu

7. Dessen Bedeutung wurde von Hirschman (1970) früh erkannt, aber in der Literatur übersehen. Englisch hieß sein Buch noch „Exit, Voice and Loyalty“, wovon in der deutschen Übersetzung (Hirschman 1974) nur „Abwanderung und Widerspruch“ übrig blieb.

8. Zum Zusammenhang zwischen Kategorisierung, Identität und Verhalten vgl. Monroe (2001). 


\section{Liliput oder Leviathan?}

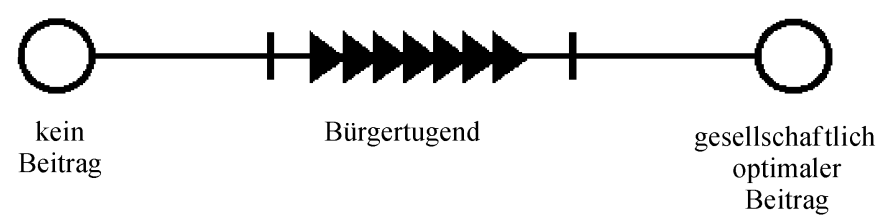

Abbildung 2 Bürgertugend und Zahlungsbereitschaft

öffentlichen Gütern animiert und potentielle Trittbrettfahrer abgeschreckt. Bürgerschaft hilft, das Problem des "second order public good“ zu überwinden und stärkt die indirekte Reziprozität (Falk und Fischbacher 2001, Bowles und Gintis 2001).

Eine Person, die diejenigen Organisationen selbst wählen kann, deren Bürger sie sein möchte, erfährt somit einen Motivationsschub. Dieser "Crowding-in" Effekt fußt auf sozialpsychologischen Prozessen und wird durch Experimente und ökonometrische Analysen in Feldstudien gestützt (Frey und Jegen 2001).

Die unmittelbare wirtschaftliche Bedeutung dieser gestärkten Bürgertugenden liegt in der Unterstützung bei der Bereitstellung öffentlicher Güter, sowie der Minderung negativer Auswirkung von Spillovers auf das öffentliche Angebot (vgl. Frey und Osterloh 2000 für das entsprechende Verhalten innerhalb Firmen). Damit wird von der Vorstellung abgewichen, bei Existenz öffentlicher Güter sei das Nash-Gleichgewicht vollständigen Trittbrettfahrens eine geeignete Erklärung des tatsächlich beobachteten Verhaltens (vgl. z.B. Ostrom 2000, Weimann 1994 oder Fehr und Schmidt 19999). Ebensowenig wird aber davon ausgegangen, öffentliche Güter würden völlig freiwillig erbracht und es käme ein Pareto-optimales Angebot zustande. Abbildung 2 illustriert, dass die Weckung der Bürgertugend eine Veränderung der Zahlungsbereitschaft für öffentliche Leistungen zwischen den Extremen keinerlei Beitrags (Nash-Gleichgewicht) und völlig freiwilligen Beitrags bewirkt.

Eine dem menschlichen Verhalten entsprechende Sicht geht somit von einem "quasi-freiwilligen Beitrag" zur Finanzierung der öffentlichen Leistungen aus. Dieser Ansatz geht von zwei Grundannahmen aus:

1. Intrinsische Motivation erklärt neben den extrinsischen Anreizen einen bedeutenden Teil des Angebots an öffentlichen Gütern. Selbst Steuernzahlen lässt sich nicht auf reinen Zwang zurückführen (vgl. Andreoni, Erard und Feinstein 1998).

2. Die institutionellen Bedingungen sind für Ausmaß und Art des intrinsisch begründeten Beitrags zu öffentlichen Gütern entscheidend. Trittbrettfahren lässt sich insbesondere dann wesentlich reduzieren, wenn drei Bedingungen erfüllt sind (vgl. Ostrom 1999, 2000):

9. In zwölf Untersuchungen liefern selbst in der letzten Runde eines wiederholten Gefangenendilemma-Spiels im Durchschnitt rund 25 Prozent aller 1042 Experimentsteilnehmer einen positiven Beitrag zum öffentlichen Gut. In einigen Experimenten sogar 46 Prozent, im Minimum immer noch 11 Prozent der Teilnehmer. Der herkömmlichen Theorie entsprechend sollten keine eigennützigen Personen in der letzten Runde etwas beitragen. 
(a) Die Individuen müssen miteinander interagieren. Diese Anforderung wird durch die spezielle Beziehung der Bürger zu „ihren“ Organisationen erfüllt.

(b) Der Prozess, der das Angebot des öffentlichen Gutes regelt, muss von den Beteiligten als gerecht angesehen werden (Tyler und Blader 2000, Frey und Stutzer 2002). Die vorgeschlagene Ausweitung des Konzeptes des Bürgers erfüllt genau diese Anforderung, denn ein Bürger hat immer Teilhabe am Entscheidungsprozess.

(c) Wer zum Nutzen aller kooperiert, darf nicht durch andere ausgenützt werden. Die quasi-freiwilligen Beiträge erfolgen nur bedingt; sie sind vor allem davon abhängig, dass sich auch andere Personen in ähnlicher Weise verhalten (vgl. Keser und van Winden 2000). Die institutionellen Bedingungen müssen deshalb so beschaffen sein, dass auch die andern Bürger zu einem Beitrag angeregt werden.

Die Möglichkeit der Individuen, selbst zu entscheiden, in welchen Organisationen sie als Bürger spezielle Rechte und Pflichten übernehmen wollen, steigert die intrinsische Motivation in Form der Bürgertugenden. Damit wird ein wesentlicher Beitrag zum Angebot öffentlicher Güter geleistet. In einer globalen Wirtschaft, in der in aller Regel Zwangsgewalt nicht wirksam angewandt werden kann, ist eine derartige Kooperationsbereitschaft besonders wichtig. Diese Bereitschaft auf der Nachfrageseite muss durch institutionelle Bedingungen auf der Angebotsseite ergänzt werden.

\section{Politisches Angebot in der globalen Welt}

Die bestehenden nationalen Grenzen sind in einer globalen Gesellschaft obsolet geworden. Die wirtschaftlichen Beziehungen werden durch die bestehenden nationalen Regelungen gehemmt und werden deshalb vermehrt umgangen.

Die Spannung zwischen den unterschiedlichen wirtschaftlichen und politischen Anforderungen lässt sich lösen, wenn von den starren Grenzen öffentlicher Körperschaften abgewichen wird: Jede staatliche Tätigkeit soll sich in dem Raum abwickeln, der dafür die geeignete Ausdehnung hat („geography of problems"). Geeignet dafür sind funktionale Körperschaften, die sich gegenseitig überlappen und die für ihre Tätigkeit notwendigen Steuern in einem demokratischen Verfahren erheben dürfen. Diese als FOCJ („Functional, Overlapping, Competing Jurisdictions“) bezeichneten Einheiten ${ }^{10}$ entstehen von unten. Gemeinden - und je nach Funktion auch einzelne Bürger - können von sich aus bestimmen, welchen funktionalen Einheiten sie beitreten wollen.

10. FOCJ sind ausführlich diskutiert in Frey und Eichenberger (1999), so dass sich eine weitere Darstellung erübrigt, vgl. auch Buchanan (1965), sowie Wehner (1992). Kritische Diskussionen finden sich z.B. bei Vanberg (2000) und Richter (2001). 


\section{Liliput oder Leviathan?}

Dieser Vorschlag flexibler staatlicher Einheiten ist somit prozessorientiert, d.h. es gibt keine fixen Lösungen, die sich technokratisch bestimmen ließen.

FOCJ brechen das Monopol des Nationalstaates auf. Es ist eine größere Zahl von politischen Körperschaften möglich, die staatliche Leistungen anbieten. Das Spektrum reicht von supra-nationalen Einheiten über den Nationalstaat (allerdings wird er nur noch dort tätig sein, wo er für Funktionen die adäquate Größe aufweist) bis zu (halb-)privaten Anbietern, die für ihre Leistungen Gebühren einfordern. Alle Einheiten sind dem politischen Wettbewerb und damit einem Effizienzdruck ausgesetzt, denn die Mitglieder können austreten, wenn sie mit den Leistungen nicht zufrieden sind und eigene FOCJ gründen. Außerdem wird die Führung der FOCJ von den Mitgliedern demokratisch gewählt und inhaltliche Probleme können durch direkte Volksabstimmungen entschieden werden. ${ }^{11}$

\section{Mannigfache Identitäten}

Führt die Möglichkeit, gleichzeitig in mehreren Organisationen Bürger zu sein und mehreren gegenseitig überlappenden öffentlichen Körperschaften anzugehören, dazu, dass man sich nirgendwo mehr zugehörig fühlt?

Diese Bedenken sind berechtigt - allerdings nur, wenn von einer traditionalen Gesellschaft ausgegangen wird. Die heutige Welt ist demgegenüber durch multiple Zugehörigkeiten geprägt. Mehrfache Loyalitäten sind ohne weiteres möglich: wir sind gleichzeitig der Region, die uns in der Jugend geprägt hat; dem Ort, in dem wir heute leben; der Firma, in der wir arbeiten; dem Sportclub, in dem wir einen Teil unserer Freizeit verbringen; dem Theater, durch das wir geistig angeregt werden, emotional verbunden. Und zu all diesen Loyalitäten kommt die nationale Zugehörigkeit hinzu.

Ein Bürger kann somit problemlos mehreren Organisationen emotional verbunden sein und entsprechende intrinsische Motivation entfalten. Eine Entsolidarisierung ist gerade nicht zu erwarten. In einer globalen Welt wäre ein Zwang, nur zu einer Organisation - meistens dem Nationalstaat - emotional verbunden zu sein, verfehlt. Die vielfachen Zugehörigkeiten in einem durch FOCJ geprägten öffentlichen Angebot entsprechen der heutigen Zeit.

\section{Abschließende Bemerkungen}

Die hier geführte Diskussion versucht den in der traditionellen Wirtschaftstheorie verankerten strikten Gegensatz zwischen "privat" und „öffentlich“

11. Wie empirische Untersuchungen für die Schweiz und die Vereinigten Staaten zur Genüge gezeigt haben, ist die Gefahr gering, dass sich politische Einheiten mit ausschließlich reichen, und solche mit ausschließlich armen Personen bilden. Vgl. Kirchgässner und Pommerehne (1996), Feld und Kirchgässner (2001). 
aufzulösen. Privat und öffentlich vermischt sich immer mehr - trotz Privatisierung und Deregulierung. So treten heute öffentliche Institutionen vermehrt in den privaten Raum ein, indem sie auf dem Markt Güter anbieten; umgekehrt bieten gewinnorientierte Firmen immer mehr öffentliche Güter an (vgl. die Evidenz in Weisbrod 2000). Früher rein interne Entscheidungen großer Firmen - etwa die Besetzung des CEO oder des CFO - sind heute infolge der Medienaufmerksamkeit öffentlich geworden. Die Gleichsetzung von „öffentlich" und "staatlich" ist weitgehend obsolet geworden.

Die Vielfalt und entsprechend notwendige Flexibilität verlangen auf Seiten der Nachfrage Bürgerschaft in vielen unterschiedlichen Organisationen und auf Seiten des Angebots multiple, überlappende demokratische Einheiten. „Der Staat" ist weder ein Liliput noch ein Leviathan, denn in der globalen Welt gibt es ihn in der herkömmlichen Form nicht mehr. Charakteristika des politischen Bereichs - Bürgertugenden in Form von Loyalität, Identifikation und Engagement - lassen sich auf wirtschaftliche Bereiche übertragen. ${ }^{12}$ Auf diese Weise kann in einer globalen Gesellschaft mit unterhöhlter staatlicher Zwangsgewalt ein über das rein Private hinausgehende Güterangebot befriedigend gelöst werden.

Die ökonomische Analyse sollte sich entsprechend von der Konzentration auf starre politische Institutionen und Beziehungen lösen. Über „Bürger eines Staates" oder die "Politik einer Nation“ zu theoretisieren (wie dies in Public Economics und der Außenwirtschaft üblich ist) kann nur noch begrenzt Einsichten liefern. Das Gleiche gilt für die Vorstellung eines „race to the bottom" der Theorie des Steuerwettbewerbs. Weil die menschliche Motivation nicht nur extrinsisch ist, ist die Entwicklung der Ökonomik in Richtung intrinsischer Anreize fruchtbar. Die Zukunft dürfte deshalb in einer motivationsund prozessorientierten Institutionenökonomik liegen.

Die Konsequenzen für die Wirtschaftspolitik sind vielfältig. Sie sollen hier für ein konkretes Problem angedeutet werden. Die zukünftige Richtung der europäischen Integration würde völlig anders aussehen, wenn eine Flexibilität hinsichtlich Bürgerschaft und staatlichen Körperschaften zugelassen würde. Die Entwicklung der Europäischen Union wäre nicht länger von der Zentrale in Brüssel und den nationalen Politikern von oben gesteuert, sondern könnte sich gemäß den Präferenzen der Individuen demokratisch und dezentral entwickeln. Die Osterweiterung könnte sich schrittweise und problemorientiert vollziehen. So könnten etwa funktionale demokratische Körperschaften zwischen politischen Einheiten in unterschiedlichen Nationen gegründet werden, die für alle Beteiligten von Vorteil sind.

12. Der „Imperialismus“ der Wirtschaftstheorie war erfolgreich (Kirchgässner 1991), aber die Zeit zum Import von Gedanken aus anderen Wissenschaften und Bereichen ist gekommen (Frey 2001). 


\section{Liliput oder Leviathan?}

\section{Literaturverzeichnis}

Akerlof, G.A. und Kranton, R.E. (2000), Economics and Identity, Quarterly Journal of Economics 115, 715-753.

Andreoni, J., Erard, B. und Feinstein, J. (1998), Tax Compliance, Journal of Economic Literature 36, 818-860.

Baldwin, R.E. und Krugman, P.R. (2001), Agglomeration, Integration and Tax Harmonization. HEI Working Paper No 1/2001. Institut Universitaire des Hautes Etudes Internationales, Genf.

Barber, B.R. (1995), Jihad vs McWorld. Random House, New York. Deutsch: Coca-Cola und Heiliger Krieg. Wie Kapitalismus und Fundamentalismus Demokratie und Freiheit abschaffen. Scherz, Bern.

Bernauer, T. (2000), Staaten im Weltmarkt. Leske und Burdrich, Opladen.

Bernholz, P. (2000), Globalisierung und Umstrukturierung der Wirtschaft: Sind sie neu? Walter Adolf Jöhr-Vorlesung 2000, Universität St. Gallen.

Berthold, N. und Naumann, M. (2001), Sozialsysteme im Wettbewerb - das Ende der Umverteilung? Wirtschaftswissenschaftliche Beiträge, Universität Würzburg.

Blankart, C.B. (2001), Öffentliche Finanzen in der Demokratie. Vahlen, München, 4. Aufl.

Blankart, C.B. (2002), Steuern als Preise, Schweizerische Zeitschrift für Volkswirtschaft und Statistik 138 (im Druck).

Bowles, S. (1998), Endogenous Preferences: the Cultural Consequences of Markets and Other Economic Institutions, Journal of Economic Perspectives 36, 75-111.

Bowles, S. und Gintis, H. (2001), The Evolution of Strong Reciprocity. Working Paper, Dept. of Economics, University of Massachusetts, Amherst.

Buchanan, J.M. (1965), An Economic Theory of Clubs, Economica 32, 1-14.

Economist (2000), The Mystery of the Vanishing Taxpayer. A Survey of Globalization and Tax. 29 January 2000.

Engel, C. und Keller, K.H. (Hrsg.) (2000), Governance of Global Networks in the Light of Differing Local Values. Nomos, Baden-Baden.

Falk, A. und Fischbacher, U. (2001), A Theory of Reciprocity. CESifo Working Paper No. 457.

Fehn, R. (2001), Ist die Globalisierung der Totengräber nationaler Sozialpolitik? Wirtschaftswissenschaftliche Beiträge, Universität Würzburg.

Fehr, E. und Gächter, S. (2000), Fairness and Retaliation: the Economics of Reciprocity, Journal of Economic Perspectives 14, 159-181.

Fehr, E. und Schmidt, K. (1999), A Theory of Fairness, Competition and Cooperation, Quarterly Journal of Economics 114, 769-816.

Feld, L.P. (2000a), Steuerwettbewerb und seine Auswirkungen auf Allokation und Distribution. Mohr Siebeck, Tübingen.

Feld, L.P. (2000b), Fiskalischer Wettbewerb und Einkommensumverteilung, Perspektiven der Wirtschaftspolitik 1, 181-198.

Feld, L.P. und Kirchgässner, G. (2001), Income Tax Competition and the State and Local Level in Switzerland, Regional Science and Urban Economics 31 (im Druck).

Frey, B.S. (2001), Inspiring Economics. Human Motivation in Political Economy. Edward Elgar, Cheltenham UK.

Frey, B.S. und Eichenberger, R. (1999), The New Democratic Federalism for Europe: Functional, Overlapping and Competing Jurisdictions. Edward Elgar, Cheltenham UK.

Frey, B.S. und Jegen, R. (2001), Motivation Crowding Theory: a Survey of Empirical 
Evidence, Journal of Economic Surveys 15, 589-611.

Frey, B.S. und Osterloh, M. (2000), Managing Motivation: Wie Sie die neue Motivationsforschung für Ihr Unternehmen nutzen können. Verlag Dr. Th. Gabler, Wiesbaden.

Frey, B.S. und Stutzer, A. (2002), Happiness and Economics: How the Economy and Institutions Affect Well-Being. Princeton University Press, Princeton NJ (im Druck).

Hirschman, A.O. (1970), Exit, Voice and Loyalty. Harvard University Press, Cambridge MA. Deutsch (1974): Abwanderung und Widerspruch. Schriften zur Kooperationsforschung, A. Studien, Band 8. Mohr Siebeck, Tübingen.

Janeba, E. (1997), International Tax Competition. Mohr Siebeck, Tübingen.

Jones, P. und Hudson, J. (2000), Civic Duty and Expressive Voting: Is Virtue its Own Reward? Kyklos 53, 3-16.

Keser, C. und van Winden, F. (2000), Conditional Cooperation and Voluntary Contributions to Public Goods, Scandinavian Journal of Economics 102, 23-39.

Kirchgässner, G. (1991), Homo Oeconomicus: Das ökonomische Modell individuellen Verhaltens und seine Anwendung in den Wirtschafts- und Sozialwissenschaften. Mohr Siebeck, Tübingen.

Kirchgässner, G. (1998), Globalisierung: Herausforderung für das 21. Jahrhundert, Aussenwirtschaft 53, 29-50.

Kirchgässner, G. und Pommerehne, W.W. (1996), Tax Harmonization and Tax Competition in the European Union: Lessons from Switzerland, Journal of Public Economics 60, 351-371.

Loewenstein, G. (2000), Emotions in Economic Theory and Economic Behavior, American Economic Review, Papers and Proceedings 90 (May), 426-432.

Monroe, K.R. (2001), Morality and a Sense of Self: The Importance of Identity and Categorization for Moral Action, American Journal of Political Science 45, 491-507.

Organ, D.W. (1988), Organizational Citizenship Behavior: the Good Citizenship Syndrome. Lexington Books, Lexington MA.

Ostrom, E. (1999), Die Verfassung der Allmende. Mohr Siebeck, Tübingen.

Ostrom, E. (2000), Collective Norms and the Evolution of Social Norms, Journal of Economic Perspectives 14, 137-158.

Richter, W.F. (2001), Institutioneller Wettbewerb und die Regelung der Zuständigkeit von Institutionen für Personen. Arbeitspapier, Universität Dortmund.

Rodrik, D. (1997), Has Globalization Gone Too Far? Institute for International Economics, Washington DC.

Rodrik, D. (1998), Why Do More Open Economies Have Bigger Governments? Journal of Political Economy 106, 997-1032.

Schaltegger, C.A. und Frey, R.L. (2001), Föderalismus zwischen dezentraler Autonomie und zentralstaatlicher Koordination, Wirtschaftsdienst 81, 176-180.

Schulze, G.G. und Ursprung, H.W. (1999), Globalization of the Economy and the Nation State, The World Economy 22, 295-352.

Siebert, H. (1998), Disziplinierung der nationalen Wirtschaftspolitik durch internationale Kapitalmobilität, in: D. Duwendag (Hrsg.), Finanzmärkte im Spannungsfeld von Globalisierung, Regulierung und Geldpolitik, Duncker und Humblot, Berlin, 41-67.

Siebert, Horst und Koop, M. (1993), Institutional Competition versus Centralization: Quo Vadis Europe? Oxford Review of Economic Policy 9, 15-30.

Sinn, H.-W. (1999), The Subsidiarity Principle and Market Failure in Systems Competition. NBER Working Paper 5411.

Straubhaar, T. (1998), Empirische Indikatoren für den Systemwettbewerb - Moderne und historische Befunde, Jahrbuch für Neue Politische Ökonomie 17, 243-272. 


\section{Liliput oder Leviathan?}

Tanzi, V. (1996), Globalization, Tax Competition and the Future of Tax Systems. IMF Working Paper 96/14, Washington DC.

Tullock, G. (1997), Trading Citizenship, Kyklos 50 (2), 251-252.

Tyler, T.R. und Blader, S.L. (2000), Cooperation in Groups: Procedural Justice, Social Identity, and Behavioral Engagement. Psychology Press, Philadelphia PA.

Vanberg, V.J. (2000), Functional Federalism: Communal or Individual Rights? Kyklos 53 (3), 363-386.

Vanberg, V. und Kerber, W. (1994), Institutional Competition among Jurisdictions: an Evolutionary Approach, Constitutional Political Economy 5, 193-219.

Van Dyne, L., Graham, J.W. und Dienesch, R.M. (1994), Organizational Citizenship Behavior: Construct Redefinition, Measurement and Validation, Journal of Management 34, 765-802.

Vaubel, R. (2000), Internationaler politischer Wettbewerb: eine europäische Wettbewerbsaufsicht für Regierungen und die empirische Evidenz, Jahrbuch für Neue Politische Ökonomie 19, 280-309.

Wehner, Burkhard (1992), Nationalstaat, Solidarstaat und Effizienzstaat. Neue Staatsgrenzen für neue Staatstypen. Wissenschaftliche Buchgesellschaft, Darmstadt.

Weimann, J. (1994), Individual Behaviour in a Free Riding Experiment, Journal of Public Economics 54, 185-200.

Weisbrod, B.A. (Hrsg.) (2000), To Profit or Not To Profit: the Dilemma of Commercializing the Non-Profit Sector. Cambridge University Press, Cambridge UK.

Wilson, J.D. (1999), Theories of Tax Competition, National Tax Journal 2, 269-304.

Abstract: Globalization is often seen to result in a smaller (Lilliput) or larger (Leviathan) state. But future public activity will be more flexible. People have multiple identities. They can be citizens of sub-and supra-national jurisdictions, semi- and non-governmental organizations and private units, even profit-oriented firms. Such attachment may be temporary, multiple or partial. To actively choose strengthens loyalty and identification, which raises the willingness to pay for publicly supplied services (in the sense of quasi-voluntary taxation). On the supply side, Functional, Overlapping and Competing Jurisdictions (FOCJ) will develop. Such flexibility of future European integration will make it successful. 